\title{
Décadrages Décadrages
}

cinéma, à travers champs Cinéma, à travers champs

13 | 2008

Anna Sanders Films, cinéma et art contemporain

\section{L'éloge spontané du chaos. Les usages de la parole dans les premiers films de Nanni Moretti}

Alain Boillat

\section{CpenEdition}

1 Journals

Édition électronique

URL : https://journals.openedition.org/decadrages/522

DOI : $10.4000 /$ decadrages.522

ISSN : 2297-5977

Éditeur

Association Décadrages

Édition imprimée

Date de publication : 10 octobre 2008

Pagination : 88-94

ISBN : 978-2-9700582-8-1

ISSN : 2235-7823

Référence électronique

Alain Boillat, «L'éloge spontané du chaos. Les usages de la parole dans les premiers films de Nanni Moretti », Décadrages [En ligne], 13 | 2008, mis en ligne le 10 octobre 2009, consulté le 03 avril 2022

URL : http://journals.openedition.org/decadrages/522 ; DOI : https://doi.org/10.4000/decadrages.522

Ce document a été généré automatiquement le 3 avril 2022.

(B) Décadrages 


\title{
L'éloge spontané du chaos. Les usages de la parole dans les premiers films de Nanni Moretti
}

\author{
Alain Boillat
}

1 La rétrospective du cinéaste Nanni Moretti présentée à Locarno s'est voulue exhaustive, allant jusqu'à intégrer ses premières réalisations en super 8 restaurées pour l'occasion - à la grande surprise du cinéaste d'ailleurs, qui semblait (ou feignait modestement) de ne pas voir l'intérêt de cette production séminale. Ne serait-ce qu'en raison de leur support, ces films, que l'on hésite à qualifier d'« œuvres » sous peine de les définir téléologiquement par rapport à un « auteur en devenir », relèvent d'une esthétique du film amateur qui marquera les premiers longs métrages du réalisateur ${ }^{1}$ et qui, sous la forme d'une mise en scène fictionnalisante de l'intime à travers une réappropriation de «genres" documentaires (le journal filmé, le film de famille), connaitra des prolongements variés dans la production ultérieure de Moretti. Plus généralement, ses tout premiers films - je m'arrêterai ici dans la filmographie de Moretti à Sogni d'oro (1981) afin de mettre l'accent sur la part la moins connue de sa production ${ }^{2}$ condensent à mon sens tous les paramètres du versant le plus intéressant du travail du cinéaste, qui se situe aux antipodes de la veine psychologisante qu'il a récemment exploitée en tant qu'acteur (Caos Calmo, Antonello Grimaldi, 2008), voire en tant que réalisateur-interprète ( La Stanza del figlio, 2001). Loin de cette «justesse de ton » que d'aucuns ont louée dans les drames récents de Moretti comme l'indice de la maturité du créateur (une réception qui s'apparente à cet égard à celle de l'œuvre de Pedro Almodovar), ses premières réalisations s'avèrent plus brouillonnes et inégales, affichant une spontanéité idiosyncrasique.

\section{Parler sans communiquer}

2 Cet autre versant, c'est celui du cinéaste bricoleur, instinctif, toujours prêt à briser la continuité de ses récits par une digression inattendue, par de brusques coups de gueule 
qui, en toute complicité avec un spectateur séduit (ou agacé), font partir en éclats le semblant de vraisemblance qu'il avait momentanément mis en place avec désinvolture. Le rythme saccadé de la juxtaposition des séquences se reflète d'ailleurs dans son travail d'acteur à travers les inflexions de la voix, où l'explication posée alterne avec la vocifération, la retenue factice avec l'explosion d'un cri de contestation. On pourrait dire que la forme même des films de Moretti prolonge l'expression orale du cinéaste qui se met en scène en tant que locuteur. En effet, ses films semblent hériter au niveau rythmique de l'intonation colorée et du débit parfois alerte de son personnage. Contrairement à d'autres cinéastes qui accordent dans certains de leurs films une place centrale à leur propre voix (Guitry, Welles, Truffaut, etc.), Moretti n'en fait pas une instance supérieure à laquelle il incomberait d'organiser le discours filmique. Dans ses premières réalisations, sa voix est significativement in ou off, rarement over ${ }^{3}$. Comme chez Woody Allen, la parole du " cinéaste », ou plutôt celle du personnage qu'il incarne (en dépit d'une part conséquente d'autobiographie), est souvent ridiculisée dans le contenu qu'elle véhicule, voire, dans les premiers Moretti, brouillée ou interrompue par d'autres sons. La parole n'endosse pas à elle seule l'appréciation émise au travers du discours filmique sur le milieu qu'il dépeint, soit chez Moretti celui de jeunes gauchistes désenchantés, mais elle est partie intégrante de ce monde. Il est à cet égard révélateur qu'en dépit du « je » affiché dans le titre du premier long métrage de Moretti (Io sono un autarchico, 1977), ce film propose un "portrait de groupe» qui permet à Moretti de railler allégrement les œuvres et la posture politico-artistique des membres d'une troupe de théâtre d'avant-garde.

«L'autarcie» de ce groupe d'amis qui s'estiment incompris se manifeste par des situations d'incommunicabilité, à l'instar des nombreuses scènes de conversations téléphoniques détournées de leur finalité communicationnelle, notamment dans les séquences récurrentes (qui ponctuent le film) où le metteur en scène de théâtre appelle depuis une cabine un critique qui, sans tenir compte de son interlocuteur, se lance dans d'interminables considérations théoriques. Dans Ecce Bombo (1978)- dont le titre énigmatique est hurlé à gorge déployée durant le générique -, les jeunes gens qui ne savent plus comment occuper leurs soirées appellent par téléphone une personne choisie au hasard pour lui faire entendre l'enregistrement d'un extrait de La Tosca de Puccini, avant de raccrocher puis de s'esclaffer de concert dans un rire puéril fédérateur. Affalés sur des sofas ou sur les chaises de bistrots, ces « vitelloni » post-68 demeurent le plus souvent plongés dans leurs pensées, amorphes. La fixité quasi constante du cadre souligne cette passivité, et met en exergue le vide qui règne après leur sortie du champ.

4 Dans Ecce Bombo, un autre personnage, extérieur à la clique d'amis, apparaît dans plusieurs séquences, se contentant à chaque fois de téléphoner à une radio locale pour faire part au monde de ses angoisses et de ses opinions politiques. L'information circule, mais personne ne la reçoit. Un journaliste de télévision intrusif et arrogant semble certes veiller à collecter des propos en organisant de façon impromptue des entretiens, mais il ne tient aucunement compte des opinions émises par les personnes interrogées qu'il rapporte catégoriquement aux lieux communs du moment.

5 Qu'elles soient déterminées par un médium technologique ou non (les micros sont très présents dans les premiers films de Moretti), les situations de conversations perturbées se déclinent de diverses manières chez le cinéaste, qui, à l'époque, n'a de cesse de dénaturaliser les interactions verbales du quotidien en les minant par la forme à la fois 
égocentrique et solitaire du monologue. Dans son cinéma, tout le monde parle, mais rares sont ceux qui se parlent, ou même écoutent les autres. Le cinéaste incarne le plus souvent un personnage qui s'exprime sur un ton péremptoire, ou alors affiche une indifférence complète à ce que l'on lui dit. Lorsqu'il s'adresse à quelqu'un, cette prise de contact s'opère fréquemment sous la forme d'une cascade de phrases interrogatives auxquelles l'interlocuteur n'a pas le temps de répondre: les questions ne sont que rhétoriques, même si leur accumulation implique souvent une remise en question du locuteur. Dans une séquence d'Ecce Bombo, Moretti va jusqu'à formuler simultanément les questions et les réponses, comme s'il ne prenait plus la peine de distribuer les répliques entre différents personnages. Les scènes de conversation téléphonique, omniprésentes dans son cinéma, constituent un lieu privilégié d'éviction de l'interlocuteur - qui est d'ailleurs souvent une interlocutrice, les échanges verbaux avec les femmes étant presque systématiquement problématiques dans ses premiers films, dominés à cet égard par une volonté de résister au sentimentalisme en le parodiant à travers une automise en scène caricaturale et exubérante, échappatoire qui vaut autant pour le personnage que pour le réalisateur. Lorsque le cinéaste figuré dans le film Sogni d'oro réfléchit au scénario qu'il est en train d'écrire et dont l'intrigue est visualisée, il s'interrompt soudainement pour constater son incapacité à concevoir une réplique pour un personnage féminin. Il n'est pas surprenant que l'ultime plan d'Ecce Bombo où semble se réaliser un rapprochement inattendu entre le protagoniste principal et une jeune femme se démarque précisément par le mutisme des deux protagonistes.

6 Il faut noter de façon plus générale que les films de Moretti, s'ils présentent une rare volubilité, n'accordent pas toujours au langage verbal une place décisive sur le plan sémantique. En effet, même si ses films sont bavards, le cinéaste ne manque jamais d'accorder la préséance à la dimension visuelle: l'acteur est un être pantomimique autant qu'un sujet parlant. Cette conception me semble prendre son origine dans les films super 8 qu'il tourna en muet et auquel il ajouta ultérieurement une parole postsynchronisée de façon assez lâche et ponctuelle. Ici, la parole in (ou plutôt déliée, qui instaure constamment un rapport de superposition artificielle à l'image) se manifeste comme un surgissement, à l'instar de l'insulte récurrente «Dadaïstes! » qui ponctue Come parli frate? (1972), parodie potache des Promessi sposi, une œuvre phare de la littérature italienne qui est transformée ici en une pantalonnade non-sensique. Le texte du roman est segmenté, redistribué selon une logique répétitive jusqu’à l'absurde ( «je jure que je jure que je jure... »), alors que les actions sont réduites à des manifestations purement instinctives, le récit " extrinsèque " au film - c'est aux spectateurs familiers de l'ouvrage "adapté » d'en reconstruire l'intrigue - reposant principalement au niveau filmique sur les gesticulations muettes de saynètes burlesques ${ }^{4}$.

7 Dans ses deux premiers films réalisés conjointement en 1973 et fonctionnant l'un par rapport à l'autre comme des vases communicants, Pâté bourgeois et La Sconfitta, Moretti post-synchronise des répliques qui ne se répondent pas, chaque personnage parlant sans interagir nullement avec son interlocuteur. Contrairement aux invités de la soirée chez les Expresso au début de Pierrot le fou (Jean-Luc Godard, 1963), un film dont l'esprit ludique et anarchisant se retrouve par moment chez Moretti ${ }^{5}$, les personnages ne débitent pas de vains slogans publicitaires, mais assument une parole qui se veut (ou plutôt se croit) personnelle: chacun s'adonne à la confession de ses obsessions névrotiques que le cinéaste, par la distance ainsi instaurée, renvoient dos à dos comme les symptômes de l'état d'une même frange de la société, la «jeunesse » composée de 
gauchistes trentenaires et oisifs à laquelle il appartient. Cette démarche qui consiste à allier un sentiment d'appartenance à une communauté - ces films-là sont avant tout réalisés «entre copains" - et une lucidité envers les clichés qu'elle véhicule et les paradoxes qui la fondent est propre à Moretti, dont l'inspiration se définit par une posture fondamentalement (auto-)ironique. L'art, la politique et la sexualité tourbillonnent dans d'interminables « discussions » qui, pour échapper au soliloque et à la vanité du stéréotype ${ }^{6}$, se muent souvent en manifestations infra-verbales, voire en violence physique. En effet, l'expression corporelle des sentiments ou des opinions constitue l'un des principaux ressorts du comique morettien.

8 Ainsi par exemple lorsque l'un des amis de son personnage évoque avec admiration le succès rencontré aux USA par Pasqualino e Settebellezze (Lina Wertmüller, 1976), un film italien contemporain mentionné à plusieurs reprises avec emportement par le personnage interprété par Moretti selon une logique du gag à répétition - un principe dont Moretti est coutumier, dans la mesure où ce procédé permet de créer un lien ténu entre des séquences ostensiblement autonomisées -, ce dernier se met littéralement à "écumer de rage »: un contrechamp nous montre son visage couvert de bave. Dans Sogni d'oro, le débat télévisé entre deux cinéastes de conviction différente vire significativement au combat de boxe. Moretti stigmatise certes ici l'abrutissement des émissions télévisées populaires, mais il utilise également ce cadre comme un prétexte au déploiement d'une vulgarité de langage et d'actions burlesques : engoncés dans un costume de pingouin, les cinéastes sont réduits à mouvoir de façon ridicule leur nageoires pour manifester physiquement leurs émotions.

\section{Nanni et Michele. Le cinéaste, le public et leur double}

Avant d'apparaître à l'écran sous son vrai nom dans Caro Diario (1993) et Aprile (1998), Nanni Moretti choisit pour les personnages qu'il incarne un patronyme qui, à l'instar des Charlot et autres Hulot, fera office d'avatar comique repris de film en film (à l'exception de La Messa è finita, 1985) : il s'agit du prénom " Michele ", auquel s'ajoutera après ses deux premiers longs métrages le nom de famille "Apicella ", qui est en réalité celui de la mère de Nanni. Certes, ce cinéaste qui n'hésite pas à donner à sa société de production (la Sacher Film) le nom d'une viennoiserie contemplée par les trois compères de Sogni d'oro dans la vitrine d'une boulangerie n'en est pas à une lubie linguistique près. Le principe de cette récurrence onomastique constitue néanmoins une caractéristique majeure du cinéma de Moretti et du lien qu'il entretient avec son public. En effet, chaque film est présenté comme une variante du précédent, le spectateur complice pouvant retrouver après quelques années l'univers personnel du cinéaste et les traits relativement stables des personnages qu'il interprète. Cette exhibition du «je » procède d'une démarche évidente d'auteurisation - les constantes attaques menées par le cinéaste sous le couvert d'un mépris gratuit contre le cinéma de grande consommation participent d'une même volonté -, et appelle également la mise en scène d'un " tu ». Dans Io sono un autarchico, les acteurs de la scène underground étaient déjà montrés dans leurs rapports avec le public à travers un débat qui tourne court, mais il n'était pas question de création cinématographique. Le passage de ce premier long métrage au suivant est principalement marqué par l'individualisation du personnage de Michele qui, dans Ecce Bombo, apparaît plus souvent indépendamment de ses acolytes, par exemple lorsqu'on le voit en slip à son domicile - une situation 
d'intimité et de mise en scène du corps du cinéaste qui radicalise une démarche déjà à l'œuvre antérieurement, notamment dans Pâté bourgeois lors des discussions dans les cabinets exigus de son appartement. Dans ce contexte de la vie privée, la question des rapports familiaux (et surtout intergénérationnels) occupe une place importante, permettant au cinéaste de nuancer le profil socio-psychologique de son personnage.

10 Si Ecce Bombo s'ouvre sur les coulisses du tournage d'un film qui n'est pas réalisé par Michele, Moretti introduit dans Sogni d'oro une dimension ouvertement réflexive, et exploite sur le plan narratif les « possibles » résultant de l'exacerbation de son ego. Ce film entremêle en une série de parallélismes et d'interférences le quotidien de Michele, réalisateur de films, avec d'une part la représentation du contenu du film qu'il scénarise puis tourne, d'autre part un ensemble continu de rêves dans lesquels il incarne un enseignant qui tombe éperdument amoureux de l'une de ses élèves. Dans chacune de ses pistes, le dédoublement se poursuit, puisque le "film dans le film " porte sur un personnage qui se prend pour Freud, et que le maitre d'école, devenu fou après le départ de sa belle, se transforme en bête, en particulier dans une scène de restaurant qui fait allusion à Dr. Jekyll et Mr. Hyde, et plus généralement via le motif de la lycanthropie associé au genre fantastique (sur un mode hallucinatoire qui n'est pas sans parentés avec Providence d'Alain Resnais, 1976, où le récit est également enchâssé dans celui de la vie du créateur). Toutefois, dans Sogni d'oro, Moretti ne met pas tant l'accent sur les sempiternelles affres de la création (même si on ne peut s'empêcher par moments de penser à Otto e mezzo, 1963, de Fellini), que sur le lien instauré avec le public. Sogni d'oro s'ouvre sur un débat réalisé à l'issue de la projection du dernier opus de Michele (un "film dans le film» dont la projection se situe hors-film, et qui se confondra avec le film lui-même), ce qui permet à Moretti de placer dans la bouche de ses protagonistes les principaux reproches adressés à son cinéma. A force de s'entendre dire qu'il s'agit d'un cinéma intellectuel qui ne ferait qu'ennuyer des gens simples tous les intervenants se réfèrent à un moment donné du film aux mêmes archétypes, « le berger des Abruzzes, le paysan de Lucanie et la ménagère de Trévise »-, Moretti les convoque à la fin en les incarnant concrètement, leur faisant prendre le train pour se réunir dans la salle où l'on discute du film. Ce pied de nez aux donneurs de leçon qui prétendent savoir quel doit être le public visé n'interdit pas à Moretti d'afficher les spécificités et les limites de son cinéma, et ainsi d'affirmer ses partis pris.

11 Sogni d'oro éveille chez son spectateur une sorte de plaisir masochiste à contempler, intégrée à l'univers diégétique, la réception négative suscitée par le film même l'expression " public de merde » reprise en chœur par les spectateurs aliénés présents sur le plateau du show télévisé relève indubitablement de l'adresse. Moretti déclarait: " Au moment du scénario [...], je me demande ce que le spectateur attend. Alors, ce qu'il attend, je ne le lui donne pas. ${ }^{7}$. Ce rapport volontairement conflictuel au public s'accompagne cependant d'une forte complicité - le public s'attend précisément à de l'inattendu - qui, dans Sogni d'oro, fonctionne également sur le mode de la «distinction » (au sens bourdieusien du terme) : le spectateur du film est rassuré de se savoir différent de ce public-là, insensible aux subtilités qu'il sait lui-même apprécier. Cette perversité de la mise en abyme morettienne n'interdit cependant pas qu'en de nombreux passages le cinéaste s'expose et provoque le rire avec un talent comique certain. On peut citer pour finir une séquence mémorable de Sogni d'oro qui condense intelligemment les particularités des films de Moretti. Invité par une chaîne de télévision à s'exprimer sur la jeunesse contemporaine - un «thème » auquel Moretti 
fut constamment associé à l'époque -, Michele se lance dans un laïus qui ne manque pas de ridicule. Après quelque temps, le caméraman, appelé par un collègue, quitte la pièce à pas de loup après avoir indiqué à Michele qu'il devait continuer de parler. Se retrouvant seul face à la caméra qui tourne, Michele est saisi d'un malaise qui nous est signifié par une suite de froids champ/contre-champs entre la machine et lui, et qui culmine dans un plan d'ensemble où il se met à crier convulsivement «Au secours !». A l'instar de cette séquence où l'exhibitionnisme fait place à la peur de n'être filmé par (et pour) personne (d'autant que le cinéaste passe lui-même devant la caméra), le cinéma de Moretti oscille entre l'exubérance délurée et le désarroi existentiel. Ce n'est pas l'un des moindres mérites de cette édition du Festival de Locarno que d'avoir permis un retour sur les premières œuvres de Nanni Moretti.

\section{NOTES}

1. Ainsi son premier long métrage Io sono un autarchico (1976) sera-t-il tourné en super 8 avant d'être gonflé en $16 \mathrm{~mm}$ en raison du succès qu'il a rencontré à sa sortie (voir à propos de la réception de ce film les informations données par Jean A. Gili, Nanni Moretti, Gremese, Rome, 2001, pp. 10-11).

2. Le film suivant, Bianca (1983), appartient sans conteste à une phase plus légitimée du travail de Moretti. Dans le champ italophone, on constate qu'un manuel d'analyse du film qui s'appuie sur le corpus filmique de Moretti débute avec Bianca (Roy Menarini, Studiare il film, I Quaderni del Battello Ebbro, Porretta Terme, 2001) et qu'une récente publication du même auteur est entièrement dédiée à ce même film (Bianca, Lindau, Torino, 2007). Jean Gili qualifie quant à lui Bianca de « mûrissement expressif ", alors que Sogni d'oro serait « réalisé par un cinéaste qui n'est peut-être pas encore parvenu à canaliser son trop-plein d'énergie » (Jean A. Gili, op. cit., p. 20 et p. 18). L'édition française de DVD qui n'avait consacré jusqu'ici aucune sortie aux premiers films du cinéaste propose depuis septembre 2008 un coffret qui comprend précisément Bianca (1984) et La Messa è finita (La Messe est finie, 1985).

3. On citera l'exception notable d'une séquence de Pâté bourgeois (1973) dans laquelle la voix over raconte une histoire stéréotypée en imitant le récitatif d'un roman-photo. Il s'agit toutefois dans ce cas d'une sorte de récit enchâssé dont la responsabilité énonciative n'incombe pas au narrateur « fondamental » du film.

4. La mutité (et respectivement l'aléatoire de la sonorisation) est exhibée dans Come parli frate? lorsque le seigneur incarné par Moretti s'étonne qu'une assiette ait pu se briser sans provoquer aucun bruit. Dans Io sono un autarchico, l'une des déclarations péremptoires du personnage de Michele sur le cinéma, parodiant la justification d'une remise en cause de l'idéologie bourgeoise telle que l'avait proposée Godard dans La Chinoise (1967) ou Vent d'est (1969), s'articule en fonction de trois termes : l'acteur est la bourgeoisie, l'image le prolétariat, et la piste son est associée à la petite bourgeoisie située entre l'un et l'autre.

5. Notons que le finale du film de Godard est cité (en noir et blanc) dans I Sovversivi (Paolo et Vittorio Taviani, 1964), film choisi par Moretti dans le cadre de sa carte blanche locarnaise.

6. En parodiant la phraséologie d'une époque et d'une communauté (les intellectuels de gauche post-68), Moretti prête une attention toute particulière à des expressions devenues vides de sens à force d'être utilisées par tous. Plusieurs niveaux du stéréotype sont convoqués dans une 
séquence de Sogni d'oro dans laquelle deux personnages secondaires expriment leur avis sur le dernier film de Don Siegel : si le premier déplore le fait que le film en question est « plein de lieux communs, de banalités, de personnages stéréotypés », le second fait l'éloge du film en arguant qu'il propose «un jeu sur les lieux communs, les banalités, les personnages stéréotypés ». La façon d'envisager le stéréotype est elle-même présentée comme un cliché.

7. Entretien figurant dans l'ouvrage de Jean A. Gili, op. cit., p. 58 (parution originelle dans Positif, $n^{\circ} 311$, janvier 1987). 